\title{
LOS ESTUDIOS DE LENGUA ÁRABE ENTRE LOS MORISCOS ARAGONESES A TRAVÉS DE LOS MANUSCRITOS DE LA JUNTA
}

Jesús Zanón*

\section{PlanteAMiento}

El objetivo del presente trabajo es el análisis de las obras gramaticales contenidas en los llamados "manuscritos de la Junta", y de todo aquel material que pudiera desvelar el sistema de aprendizaje de la lengua árabe entre los moriscos de Almonacid de la Sierra, o, por extensión, de los moriscos aragoneses. Con este análisis tratamos de extraer respuestas acerca de la función y las características de las obras gramaticales, dentro del contexto, más general, de las obras conservadas en árabe.

Se sabe bien que la situación lingüística de mudéjares y moriscos no era homogénea1. Si los mudéjares y moriscos valencianos y granadinos mantuvieron de modo general el árabe hablado y escrito, los mudéjares y moriscos castellanos y aragoneses perdieron pronto su dialecto, pero conservaron la lengua literal parcialmente, destinándola fundamentalmente a usos religiosos y jurídicos; para otras manifestaciones literarias emplearon sobre todo la lengua castellana, que escribían normalmente en caracteres árabes (aljamiado). En este contexto lingüístico se encuadran los manuscritos árabes y aljamiados hallados en Almonacid de la Sierra en 1884, y adquiridos, posteriormente, por la Biblioteca de la Junta para Ampliación de Estudios².

Universidad de Alicante.

1. Véase M" Jesús Viguera, «Prólogo» a F. CORRIEnTE CóndobA, Relatos píos y profanos del ms. aljamiado de Urrea de Jalón, Institución Fernando el Católico, Zaragoza, 1990, pp. 16-22.

2. Posteriormente fueron depositados en la Escuela de Estudios Árabes de Madrid, heredera de la sección de árabe del Centro de Estudios Históricos de la Junta para Ampliación de Estudios e Investigaciones Científicas. Tras la guerra civil, la Escuela fue integrada en el C.S.I.C., que ocupó el lugar de la Junta, y se denominó Instituto Miguel Asín. Actualmente ocupa su lugar el Departamento de Estudios Árabes e Islámicos del Instituto de Filología del C.S.I.C. De aquí que los manuscritos hallados en Almonacid de la Sierra se hayan denominado sucesivamente -o indistintamente- "manuscritos de la Junta", "de la Escuela de Estudios Árabes" o "del Instituto Miguel Asín". En el presente trabajo utilizaremos su pri- 
Las ideas y conclusiones de este trabajo se construirán sobre la siguiente premisa: los manuscritos de la Junta pueden considerarse, en su conjunto, como una muestra aleatoria de los libros en poder de una comunidad morisca aragonesa. No creo que sea muy necesario argumentar o discutir esta idea de principio en el estado actual de las investigaciones sobre los moriscos aragoneses. No obstante se puede alegar lo siguiente:

- Se trata de una muestra, porque el número de obras conservadas es suficiente para reconocer qué tipo de disciplinas interesaban a sus propietarios $\mathrm{y}$ en qué medida.

- Probablemente es una muestra aleatoria, porque la acción de esconder los libros y papeles escritos en árabe o aljamiado para salvarlos del fuego de la Inquisición, sin que existan indicios de una previa selección de su contenido, podría considerarse un hecho aleatorio o azaroso, como sin duda lo es la destrucción de una parte de los códices a manos de los muchachos de Almonacid "que se entretuvieron en romper las hojas y en hacer hogueras con ellas, destrozando más de ochenta volúmenes y quemando, quizá por completo, no pocos" ${ }^{\prime \prime}$.

- Muestra aleatoria de los libros y escritos en poder de una comunidad morisca aragonesa, porque de la gran variedad de sus contenidos, desde la culta teología de Algazel hasta los populares amuletos, se deduce que los manuscritos ocultados no pudieron pertenecer a un solo propietario o a una sola familia.

El análisis cuantitativo de las obras halladas ocultas en Almonacid nos permitirá saber en qué medida interesaba la gramática árabe a la comunidad morisca de esa localidad, o, si puede extrapolarse, a las comunidades moriscas de Aragón. De paso nos permitirá saber en qué medida eran estudiadas y transmitidas otras disciplinas islámicas y, también, la relación numérica entre escritos árabes y aljamiados ${ }^{4}$.

Para ello se ha tomado como base del cómputo el número de líneas escritas en los manuscritos, siguiendo los datos ofrecidos en el catálogo dirigido por J. Ribera y M. Asín ${ }^{5}$, diferenciando, por un lado, texto árabe y texto aljamiado, y, por otro, las diversas disciplinas contenidas en los textos árabes.

mera denominación, siguiendo el catálogo dirigido por Julián Ribera y Miguel Asín y realizado por ellos mismos y por Maximiliano Alarcón, Ambrosio Huici Miranda y Cándido González (y la colaboración de Tomás Navarro Tomás). Cfr. Manuscritos árabes y aljamiados de la Biblioteca de la Junta, Madrid, 1912.

3. F. CODERA, «Almacén de un librero morisco descubierto en Almonacid de la Sierra», Boletín de la Real Academia de la Historia, Madrid, V (1884), apud Manuscritos árabes y aljamiados..., p. VI.

4. La idea de realizar este cómputo fue sugerida por la prof. $\mathrm{M}^{\mathrm{a}}$ Jesús Rubiera en el curso de un seminario preparatorio del presente Simposio en el área de Estudios Árabes e Islámicos de la Universidad de Alicante.

5. Manuscritos árabes y aljamiados... 


\section{TEXTOS ALJAMIADOS Y TEXTOS ÁRABES}

Del cómputo realizado se desprende que los textos escritos en aljamiado constituyen el 62,4\% del total de los manuscritos de la Junta, y los escritos en árabe el restante $37,6 \%$. Creo que este resultado no sorprenderá a aquellos investigadores de la literatura aljamiada familiarizados con el catálogo y habituados a estudiar sus manuscritos o las ediciones de éstos. Pero quizá no sea ocioso observar que la mayor parte de los manuscritos en lengua árabe está todavía sin estudiar.

\section{DISTRIBUCIÓN POR MATERIAS DE LOS TEXTOS ÁRABES}

Como se ha referido anteriormente, he realizado el cómputo de los textos árabes por materias con el objetivo fundamental de sopesar en qué medida los moriscos aragoneses valoraban los estudios gramaticales frente a las otras disciplinas. Los resultados son los siguientes.

En primer lugar, la disciplina más representada es el derecho (figh), con un $33,8 \%$ sobre el total de textos árabes. Puede anotarse, además, que dentro de los textos jurídicos, el 71,6\% lo constituyen obras de doctrina mālikí; el resto de las obras jurídicas son "formularios notariales" (watā'iq) $=12,1 \%$; "formularios de redacción de contratos" ('aqd al-šsuru $\bar{t}$ ) $=11 \%$, y "cuestiones resueltas" (masă'il)=5,3\%. Debemos observar, por tanto, que las obras de tipo jurídico de los moriscos de Almonacid son de indudable carácter práctico.

La segunda disciplina más representada desde el punto de vista cuantitativo es el Corán (= 20,4\% del total). El 73,1\% de los textos coránicos son azoras, y el $26,9 \%$ comentarios (tafșît).

Si agrupamos gramática y lexicografía en una sola disciplina de estudios filológicos, ésta ocupa el tercer lugar en extensión, con un $16,6 \%$ del total. El porcentaje de gramática y de lexicografía es aproximadamente el mismo ( $48,7 \%$ y $51,3 \%$ respectivamente).

Es decir, el $60,8 \%$ de los textos árabes ocultados en Almonacid de la Sierra pertenecen a una de estas tres disciplinas: derecho, corán o filología. El resto de las disciplinas, por orden decreciente de representatividad, es el siguiente:

$A d a b=11,8 \%$. Debo precisar que he considerado adab cualquier texto narrativo, si bien prevalece el de tipo piadoso ${ }^{6}$.

Mística $=4,4 \%$

Hadi $\underline{t}=3,8 \%$. De este conjunto de textos, los repertorios de hadit $\underline{\text { tes ocu- }}$ pan un $79,5 \%$ y las obras de crítica interna o ușull al-hadit t, un $20,5 \%$.

6. Sobre el $a d a b$ de tipo religioso en al-Andalus véase $\mathrm{M}^{\mathrm{a}}$ Jesús RuBIERA, Literatura Hispanoárabe, 1992, pp. 184-186. 


$$
\begin{aligned}
& \text { Jutbas }=3,7 \% \\
& \text { Teología }=3 \% 7 \\
& \text { Oraciones }=2,3 \% \\
& \text { Poesía }=0,2 \%
\end{aligned}
$$

\section{RETRATO DEL MORISCO LETRADO}

A partir de los datos anteriores se podría, quizá, realizar un "retrato" del morisco aragonés letrado ${ }^{8}$.

Sería, en primer lugar, un alfaquí, naturalmente mālikí, preocupado por mantener, transmitir y observar las tradiciones jurídicas islámicas en la lengua del Corán. Es un alfaquí enemigo de profundizaciones especulativas, que se sirve de "manuales de consulta rápida", como el Mujtasar de alTulaytulì, pero no parece capacitado o interesado en estudiar las fuentes clásicas del derecho mālikí o, al menos, en la muestra no han aparecido fragmentos del Muwatta' de Mālik o de la Mudawwwana de Sahnūn, obras básicas en la formación dë cualquier alfaquí de al-Andalus desde la recepción de la escuela mālikí. El alfaquí morisco aragonés se sirve de modelos árabes para la redacción de todo tipo de documentos legales, y utiliza las opiniones dadas por los juristas del pasado a través de las colecciones de masá'il. Los textos jurídicos son esencialmente prácticos y posibilitarían que el alfaquí llevara a cabo sus funciones en el seno de su comunidad.

En segundo lugar, el morisco aragonés letrado es un mu allim o maestro de escuela coránica, y un lector coránico o almocrí. El Corán es, obviamente, el texto fundamental del Islam, que desde un punto de vista ritual sólo puede utilizarse en árabe. Es también primera fuente de derecho y modelo lingüístico. Es, finalmente, el texto básico de lectura y memorización en los primeros estudios o estudios primarios de una sociedad islámica tradicional. Por todo ello no es de extrañar que, entre los textos ocultados en Almonacid, el 15\% de los textos árabes conservados sean aleyas del Corán. Puede justificarse esta abundancia de aleyas por su triple función religiosa, ritual y pedagógica. Por otro lado, a través de un manuscrito aljamiado ${ }^{9}$, sabemos que se mantenía el modo de lectura del medinés Nāfi', una de las variantes canónicas y la más difundida sin duda en al-Andalus ${ }^{10}$.

7. Se trata, en concreto del resumen de la obra de al-GAZĀLT, Ihy̆ă’ 'ulūm al-dīn.

8. Tendremos exclusivamente en cuenta las obras escritas en árabe. Para una sintesis sobre la sociología de la literatura aljamiado-morisca véase Míkel de EPALZA, «Introducción» a Luis F. BERNABÉ PONS, El cántico islámico del morisco hispanotunecino Taybili, Zaragoza, 1988, pp. 526. Véase también Míkel de EpalzA, Los moriscos antes y después de la expulsión, Madrid, 1992.

9. En concreto, el manuscrito número XII, ff. 1-157.

10. Véase J. ZANON, La vida intelectual en al-Andalus durante la época almohade: Estudio de la «Takmila» de Ibn al-Abbār, Tesis Doctoral inédita. Sobre la lectura de Nāfi puede consultarse 
En la cultura musulmana las ciencias filológicas ocuparon un papel importante entre las disciplinas islámicas (evidentemente también entre las profanas). Téngase en cuenta que una de las causas principales del desarrollo de los estudios filológicos fue el Corán, que, como palabra de Dios, se constituyó en modelo y regla de la lengua árabe ${ }^{11}$. El morisco aragonés, que había sustituido como lengua materna el árabe hablado por el castellano, debía hacer un esfuerzo mucho mayor que sus correligionarios valencianos y granadinos para aprender la lengua árabe literal. La posesión de gramáticas y diccionarios era fundamental para sus comunidades, y ello queda reflejado en el alto porcentaje de este tipo de obras en la muestra de las obras de Almonacid. Desarrollaremos más adelante este punto.

Las ciencias islámicas, es decir, aquéllas que nacieron como resultado de la expansión del Islam, son fundamentalmente de tres tipos: coránicas, jurídicas y las relacionadas con la tradición del Profeta o hadît. Esta última está muy poco representada entre los libros de Almonacid (3,8\% del total). Creo que ello tiene una fácil explicación: el estudio del hadi $\underline{t}$ no tenía ninguna aplicación práctica, ritual o jurídica en las comunidades moriscas. Hacía ya varios siglos que el hadit se había codificado, y que su transmisión consistía en una labor casi meramente erudita o memorística. De aquí que desde el siglo XI comenzaron a proliferar colecciones de fácil memorización, como las colecciones de "cuarenta hadit t-es", los "hadit t-es encadenados" o aquéllas en las que se prescinde deliberadamente de las cadenas de transmisión, como el $\breve{S} i \bar{\imath} \bar{b}$ de al-Qudā $\grave{i}$, que aparece entre los libros de Almonacid ${ }^{12}$.

En definitiva, las obras escritas en árabe de los manuscritos de la Junta presuponen que el segmento más culto de la sociedad morisca busca mantener a través de la lengua árabe tres órdenes de la vida de aplicación práctica:

En primer lugar la legalidad jurídica; en segundo lugar el ritual religioso, a través del Corán, pero también mediante las colecciones de oraciones y de sermones (jutba-s). En tercer lugar se busca mantener el sentimiento religioso, que entrevemos por las colecciones de narraciones sobre los profetas, el resumen de la obra de al-Gazālì Ihyă ‘ulum al-dìn y las obras de mística.

Una comunidad musulmana dentro de un estado islámico mantendría otros géneros de producción escrita que los moriscos aragoneses no conservan, o apenas mantienen. Así, son superfluas la poesía, la prosa ornada, la producción bio-bibliográfica, y las obras de especulación y de erudición. Son superfluas porque no tienen aplicaciones prácticas dentro de una comunidad marginada y de pocos recursos económicos e intelectuales.

Fijémonos en lo que supone la ausencia de obras bio-bibliográficas en las comunidades moriscas. En una sociedad islámica tradicional, como la an-

R. BLACHÈRE, Introduction au Coran, París, 1947, pp. 118-119. Sobre las lecturas coránicas en el occidente islámico véase SA 'ĪD A 'RĀB, Al-qurrá' wal-qiră'ât bi-l-Magrib, Beirut, 1990.

11. Véase Henri FleISCH, Traité de philologie arabe, vol. I, Beirut, 1961, p. 2.

12. Manuscrito $\mathrm{n}^{\circ} \mathrm{XXIX}, \mathrm{y}$ fragmentariamente en $\mathrm{n}^{\circ} \mathrm{XXXIX}, \mathrm{ff} .1-24$. Esta obra fue muy difundida en al-Andalus durante la época almohade. Véase J. ZANÓN, op. cit. pp. 156-158. 
dalusí, la base de la transmisión de la ciencia es el ulema, maestro en una o varias disciplinas, que enseña obras a sus discípulos siguiendo determinadas líneas de transmisión o riwâya-s, de valor ponderable, y utilizando distintos métodos o formas de transmisión ${ }^{13}$. La actividad de los ulemas tiene su reflejo en la literatura bio-bibliográfica, que es inseparable del proceso de producción y transmisión de las ciencias islámicas; podría decirse que es su vademécum. Los moriscos ni conservan ni producen este tipo de literatura, porque el sistema de transmisión de la ciencia aludido anteriormente no es operativo en sus comunidades. No se puede, por tanto, hablar de "escuelas" entre los moriscos. El proceso de producción intelectual en árabe se ha agotado, y el proceso de transmisión a duras penas se mantiene bajo formas, probablemente, muy elementales.

\section{EL APRENDIZAJE DE LA LENGUA ÁRABE ENTRE LOS MORISCOS DE ARAGÓN. OBRAS GRAMATICALES Y LEXICOGRÁFICAS Y MATERIALES AFINES}

El objeto central del presente estudio era el análisis de los materiales gramaticales y lexicográficos que se encuentran entre los manuscritos de la Junta. Era necesario, previamente, sopesar estos materiales en el conjunto de los escritos árabes conservados. Del análisis global han llamado la atención dos aspectos:

1) Existe una economía de obras en árabe. Solamente encontramos aquéllas que son esenciales para el mantenimiento de la tradición islámica desde el punto de vista del ritual religioso (aleyas coránicas, oraciones y sermones rituales), del mantenimiento del sentimiento religioso (narraciones piadosas y algunos escritos místicos y teológicos) y de la legalidad (escritos jurídicos).

2) Teniendo en cuenta que la lengua de los moriscos aragoneses era el castellano, los materiales gramaticales y lexicográficos serían imprescindibles para que el mantenimiento de la lengua árabe en el ritual y en las prácticas legales fuera efectivo. Luego puede deducirse una función marcadamente auxiliar y práctica.

Desde este punto de vista vamos a considerar qué es lo que se conserva y el modo en que podría utilizarse dentro del proceso de aprendizaje y de mantenimiento del árabe como lengua vehicular literal.

\section{EL ALFABETO}

El primer obstáculo es evidentemente la grafía, escollo que se supera rápidamente mediante ejercicios de escritura, como los que aparecen entre

13. Sobre este último punto véase J. ZANÓN, «Formas de la transmisión del saber islámico a través de la Takmila de Ibn al-Abbār», Sharq al-Andalus, 9, 1992, pp. 129-149. 
los legajos de Almonacid. En concreto, se encuentran catalogados en tres lugares diferentes ejercicios caligráficos en caracteres árabes ${ }^{14}$. También se conservan ejercicios de escritura en caracteres latinos ${ }^{15}$.

Pero además encontramos, en dos breves escritos redactados en castellano, el sistema de equivalencias fonéticas de las letras del alfabeto árabe y las reglas de lectura y de escritura. No podían estar redactados en aljamiado, cuando se trata de enseñar a principiantes castellanoparlantes los primeros pasos en el aprendizaje de la lengua árabe ${ }^{16}$.

Tenemos, pues, documentado entre los manuscritos hallados en Almonacid la descripción teórica del sistema grafológico del árabe y una serie de ejercicios prácticos.

\section{LA GRAMÁTICA}

El segundo paso en el aprendizaje de la lengua, siguiendo un método tradicional, consiste en la asimilación de las reglas morfológicas y sintácticas. Como en el caso de la grafología, hallamos entre los manuscritos de Almonacid obras de carácter teórico, pero también una serie de folios de ejercicios gramaticales.

La relación de obras gramaticales es la siguiente:

- Kitāb tabsirat al-mubtadī wa-tadkirat al-muntahī, de Abū Muhammad ‘Abd Allāh b. `Alī b. Ishạa al-Ṣaymarī. Autor iraquí fallecido en $541 / 1146^{17}$.

14. Que son los siguientes:

- En LXIV, 4) "adornos caligráficos, como ejercicios de escritura".

- En LXXXIX, folios procedentes del manuscrito XV, se encuentran doce papeles en árabe y aljamiado en donde, entre otros asuntos, hay ejercicios de escritura.

- En LXXX, folios procedentes de las tapas del manuscrito XVI, se encuentran "ocho papeles (...) algunos con ensayos de dibujos caligráficos".

15. En concreto aparecen los siguientes:

- IV, guardas (vide Homenaje a D. Francisco Codera, Zaragoza, 1904).

- En LXVI, folios procedentes de las tapas del manuscrito I se encuentran "diez folios apolillados, lengua castellana, letra española, siglo XVI; contienen ejercicios caligráficos".

- En LXXV, folios procedentes de las tapas del manuscrito XI, se encuentran "cuatro papeles muy destruidos, en castellano, casi ilegibles; letra del siglo XV, de manos diferentes y sin aparente sentido, como ejercicios de escritura".

- En LXXXII, folios procedentes de las tapas del manuscrito XVIII, se encuentran "ocho folios en castellano, con ejercicios caligráficos".

16. En concreto:

- LX, fol. 119v-122: "La regla de la lienda del Alcorán. Dictada por cuatro sabios para que pudiesen leer el libro santo las gentes de lenguas apartadas del Alcorán. Es una explicación muy confusa, acerca del uso y valor de las letras y signos auxiliares de la escritura".

- En XCVIII, folios procedentes de las tapas del manuscrito XXXIV se encuentran dos folios escritos en castellano que "tratan de la equivalencia fonética de los signos árabes; letra del siglo XVI". A continuación se transcribe su contenido. Sin duda es una traducción literal de una capítulo dedicado a la fonética de alguna gramática árabe.

17. J-XV, 143 folios escritos. BROCKELMANN, G.A.L., I, pp. 280; KAHHÄLA, Mu'ŷam al-mu'allifïn, VI, p. 87. 
- Kitäb al-ŷmal fi l-nahw, del gramático de Basora Abū l-Qāsim `Abd alRahmān b. Ishạa al-Zaŷỹâỹi (m. 337/949), obra muy difundida en alAndalus $^{18}$.

- Šarh Kitāb al-ŷyrrūmiyya, de Abū Ishāq Ibrāhīm b. Gālib al-Ansāāĩ. Se trata de un comentario de 34 folios del Kitāab al- $\hat{y} u r r \bar{u} m i y y a^{19}$. Tiene traducción interlineal aljamiada parcial.

- Kitāb al-ŷurrūmiyya, con traducción aljamiada interlineal, de Abū `Abd Allāh Muhammad b. Dă'ūd b. Ầurrūm al-Ṣinhāŷî (m. en Fez en 722/1322), obra tardía pero muy difundida ${ }^{20}$.

- Kitāb al-šâyara fí l-nahw wa-huwa l-musammà bi-Kitāb al-taqrīb, del gramático de Bagdad Abū Ishạq Ibrāhīm b. Sahl b. al-Sarrī al-Zaŷŷâŷ al-Nahịwì (230/844-311/923), con traducción aljamiada interlineal ${ }^{21}$.

- Fragmento de gramática árabe no identificada, de 16 folios $^{22}$.

Además se encuentra un fragmento en escritura aljamiada que es una traducción literal del Kitāb al-ŷyrrūmiyya $a^{23}$.

Junto a estas obras de carácter teórico, hallamos, en dos lugares distintos, papeles de ejercicios prácticos ${ }^{24}$.

Entre las obras gramaticales de Almonacid quizá pueda extrañar la ausencia de la obra gramatical considerada definitiva en el mundo árabe, y muy estudiada y difundida en al-Andalus: el Kitāb de Sĩbawayhi (m. hacia 180-194/796-809). En lugar de este clásico gramatical encontramos obras más breves y simplificadas, que se prestan mejor a la divulgación, como el Kitāb

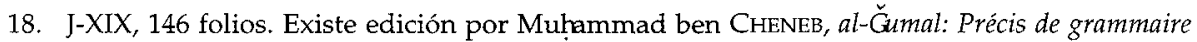
arabe, París, 1957. Véase BrockelMANN, I', 110, 122.

19. J-LIX, ff. $104 \mathrm{v}-137$.

20. J-LIX, ff. 138v-175. Véase BrockeLMANin, II, 308-10 y SII, 332-5; E.I.2, III, 719 (G. Troupeau, «Trois traductions latines de la "Muqaddima" d'Ibn Ağurrüm», en Études dédiées à la memoire de E. Lévi-Provençal, I, París, 1962, pp. 359-365.

21. J-LIX, ff. 176-216. Sezgin, GAS, IV, pp. 81-82; pero este mismo autor interpreta, creemos que con acierto, en op. cit., p. 82 nota 1, y p. 169, que la obra citada se trata en realidad del Kitáb Ibn al-Šâyara, es decir, del libro de Abū Bakr Aḥmad b. Kāmil b. Šaŷara al-Qãdī (m. 350/961), una de cuyas obras de gramática se titula Kitäb al-Tagrīb fi kašfal-garĭb.

22. J-XXXVII, ff. 166-181. Hay también fragmentos de gramática más breves en el mismo ms. XXXVII, ff. $38 \mathrm{v}-41$, y en III, ff. $228 \mathrm{v} ;$ XXV, f. $1 \mathrm{r}$ de las guardas. En las carpetas de folios sueltos hay fragmentos gramaticales en: LXIV (9); LXXX (4); LXXXVI (1); CI D(8).

23. J-XII, ff. 160-189.

24. En concreto:

- En XCVIII, folios procedentes de las tapas del manuscrito XXXIV, se encuentra un folio "de ejercicios gramaticales".

- En CI, papeles sueltos procedentes de diversos códices, se encuentran "catorce papeles de borrador, conteniendo ejercicios caligráficos, gramaticales y lexicográficos; algunos de ellos parecen ensayos de lectura y escritura del alfabeto árabe; otros son apuntes preparatorios de versión aljamiada de textos árabes; uno de ellos contiene tanteos de dibujo caligráfico". 


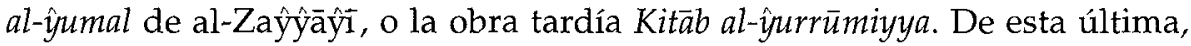
muy concisa, dice G. Troupeau que era fácil de aprender de memoria. Gozó de gran reputación en todos los países árabes hasta nuestros días, y suscitó unos sesenta comentarios, lo que da idea de su gran aceptación y difusión ${ }^{25}$. Podría pensarse que el Kitāb de Sïbawayhi se perdería, junto con otras obras, alimentando el fuego, no ya de la Inquisición al que parecían destinadas, sino el de las hogueras de la chiquillería de Almonacid. Pero, siendo así, sería una casualidad que también acabasen en la hoguera las obras máximas de cada una de las disciplinas islámicas, puesto que en derecho no se ha conservado ni el Muwattá de Mālik ni la Mudawwana de Sahnūn, pero sí algunos resúmenes de carácter divulgativo, como el Mujtasar de al-Tulaytulī. O en tradiciones proféticas no se ha conservado ninguna de las colecciones canónicas, especialmente las de al-Bujārì, Muslim y al-Tirmid̄î, que fueron las más difundidas en al-Andalus; y en cambio ha llegado a nosotros el Šihāb de al-Qudāì colección tardía y de fácil memorización. En consecuencia, hay que insistir en que las obras en árabe conservadas y transmitidas por los moriscos aragoneses son, en general, concisas y de fácil memorización. Los moriscos no utilizaban, excepto el Corán, sus "fuentes clásicas", sino algunas de sus posteriores adaptaciones, resúmenes o compendios de menos espesor.

\section{LA LEXICOGRAFIA}

Entre los manuscritos de Almonacid encontramos además materiales lexicográficos, en concreto dos fragmentos del Mujtasar o resumen del Kitāb al-`ayn, del sevillano Abū Bakr Muhammad b. Hasan al-Zubaydì (m. $379 / 985)^{26}$.

\section{OTROS MATERIALES: LAS TRADUCCIONES INTERLINEALES}

Hasta aquí hemos revisado aquellas obras y materiales de carácter gramatical evidente. Sin embargo, cualquiera que se haya enfrentado al proceso de aprendizaje de la lengua árabe echaría en falta, al menos, materiales lexicográficos más accesibles que el citado diccionario árabe-árabe de alZubaydi. El enorme y complejo caudal léxico del árabe hace que esta lengua sea inasequible sin glosarios y diccionarios adecuados. ¿Cómo podrían salvar este obstáculo los moriscos castellanoparlantes que se iniciaban en el estudio de los textos árabes? ¿O sería insalvable?

25. $\mathrm{EI}^{2}, \mathrm{III}, 719$.

26. J-XXXV, 145 folios escritos y J-XLIX, 137 folios escritos. Además, en la carpeta número $\mathrm{CI}$, legajo de papeles sueltos procedentes de diversos códices, se encuentra un fragmento que "parece de un diccionario de homónimos árabes". Para la biografía de al-Zubaydĩ véase J. PÉREZ LÁZARO, «Una obra de Ibn Suhayd sobre lahn al-'ämma en al-Andalus», Al-Qantara, VII, 1986, p. 253, nota 2 . 
Julián Ribera, tras examinar los manuscritos de Almonacid, declaraba lo siguiente:

"Las traducciones aljamiadas hechas por los moriscos aragoneses indican, en la inmensa mayoría de los casos, que son arabistas pésimos, incapaces de entender con alguna claridad el árabe: son peores -concluía- que los peores de los más malos arabistas actuales" ${ }^{\prime 27}$.

J. Ribera debía referirse, sin duda, no a cualquier traducción, sino a las traducciones estrictamente literales, que se encuentran generalmente escritas de forma interlineal entre las obras árabes. Este tipo de versiones han sido estudiadas desde un punto de vista formal por Ben Jemia ${ }^{28}$, quien las denomina "aljamía calco", porque, efectivamente, las traducciones interlineales son un calco, palabra por palabra, del texto en árabe, o, como expresa un autor morisco en el título de cierta obra, están puestas "de arabio en romançe palabra despues palabra" 29 .

Si leyeramos una traducción en "aljamía calco" sin conocimiento de las particularidades sintácticas de la lengua árabe, dicha traducción sería para nosotros del todo ininteligible. Lo mismo le sucedería a un morisco que ignorara la lengua árabe ${ }^{30}$. De lo que se desprende que la traducción en "aljamía calco" no podía tener la función de sustituir el texto árabe, como hoy en día tiene cualquier traducción. Tampoco podemos suponer que los moriscos derrocharan tanto tiempo y energías en realizar una labor inútil.

La clave de la función de este tipo de versiones nos la da su misma inclusión interlineal: cada palabra árabe tiene justamente debajo su traducción en aljamiado. Está claro que no se respeta la sintaxis del español, pero lo que se pretende es dar la explicación de cada una de las palabras, y en su mismo orden, del texto árabe al modo de determinadas glosas interlineales castellanas que aparecen en ciertos códices latinos medievales. Lo fundamental es el texto árabe, que se ha de comprender y memorizar, siguiendo el sistema de enseñanza tradicional. El texto interlineal aljamiado sólo tiene sentido si al mismo tiempo se lee el texto árabe. El texto aljamiado ayuda a comprender el texto árabe que se está memorizando.

27. Manuscritos árabes y aljamiados..., p. xxiii.

28. Véase "Lengua morisca y aljamía calco», en TEMIMI, Actes de la prémière Table Ronde du C.I.E.M. surr la littérature aljamiado-morisque: hybridisme linguistique et univers discursif, Túnez, Centre de Recherches en Bibliothéconomie et Sciences de l'Information, 1986, pp. 12-26. Y la monografía La langue des derniers musulmans de l'Espagne, Publications de l'Úniversité de Tunis, 1987.

29. El título completo es: "Exte ex el xarhe de la riçala y su declaraçion puesta de arabio en romançe palabra despues palabra"; véase Manuscritos árabes y aljamiados..., p. 266.

30. La hipótesis de Ben Jemia es que el calco sintáctico y estilístico se produce a causa de que el árabe y su prestigio cultural es un modelo ideal y subconsciente. No ofrece argumentos que apoyen esta idea (véase La langue..., pp. 128-129). 
El sentido de las traducciones interlineales sería, por tanto, el de aportar la clave léxica del texto árabe; tendrían, pues, la función de glosarios. Servirían al mismo tiempo para mostrar el sistema sintáctico del árabe. Tendrían, en definitiva, una función pedagógica.

Por otro lado, la falta de materiales léxicos que hemos observado entre los manuscritos de Almonacid no sería tal. Los glosarios moriscos serían cada una de las traducciones aljamiadas interlineales.

Esta interpretación encajaría, en cierto modo, en la tradición de glosas medievales de la España cristiana, aspecto estudiado por M. Díaz y Díaz ${ }^{31}$.

Puede observarse, finalmente, que los textos con traducción interlineal, que tal vez podríamos rebautizar de glosas interlineales, son obras de carácter culto y de carácter islámico - no encontramos ningún texto de literatura popular árabe con traducción interlineal en aljamía- es decir, todas las obras con traducción interlineal son básicas para la formación en alguna de las disciplinas islámicas. Lo que nos lleva de nuevo a insistir en el carácter puramente pedagógico de las traducciones en aljamía calco.

\section{CONCLUSIONES}

Las obras halladas en Almonacid pueden considerarse una muestra representativa de la actividad intelectual de los moriscos aragoneses. Por tanto, el análisis global o particular de estas obras nos permite hallar respuestas, unas cuantificables, otras no, al problema de la conformación de su vida intelectual $y$, dentro de ella, a su específica situación lingüística.

La situación lingüística de los moriscos aragoneses es el eje de este trabajo, enfocado sobre la función de la transmisión de obras gramaticales y sus características. Éstas no pueden estudiarse separadamente del resto de las obras conservadas en árabe, pues todas juntas forman una unidad.

La comunidad morisca castellanoparlante de Almonacid conservaba, a comienzos del siglo XVII, un importante número de obras en árabe que hemos calculado en torno al 37,5\% del conjunto de sus libros. Excepto el Corán, no poseían las obras clásicas de las disciplinas islámicas fundamentales -incluida la gramática-, sino compendios y resúmenes de fácil utilización y memorización. Por otro lado, las obras en árabe se circunscriben fun-

31. Véase Las primeras glosas hispánicas, Barcelona, 1978, y Libros y librerías en La Rioja altomedieval, Logroño, 1991 ( ${ }^{\mathrm{a}}$ ed.), de donde destacamos la siguiente cita (p. 106, nota 33): "El mecanismo glosístico se desarrolla especialmente en la región de Burgos-Rioja, desde comienzos del siglo XI sobre todo, y acaso como consecuencia no tanto de las necesidades de la predicación, según suele decirse, sino dentro de la actividad escolar. Las glosas y glosarios, elementos básicos de la cultura de estos siglos, se emplean como un medio más de formación, y no el de menor rango. Esta formación, ya muy romanceada, no distingue entre glosas latinas, frecuentísimas, y glosas románicas, progresivamente abundantes". 
damentalmente a aquéllas que posibilitan el mantenimiento de la lengua del Corán en la vida jurídica y religiosa. Son obras exentas de complejidad, prácticas y de disciplinas limitadas. No obstante la lengua haría infranqueable su comprensión, si no fuera por el mantenimiento en la comunidad de gramáticas y diccionarios.

Es manifiesto el carácter práctico de los estudios gramaticales. El primer contacto con la lengua árabe se hace en castellano, lengua en la que se explica todo el sistema grafológico. Parte de las gramáticas, sencillas y fáciles de memorizar, vienen acompañadas de su traducción aljamiada. En algunos folios se encuentran además ejercicios de escritura y de gramática.

El sistema de traducción interlineal que encontramos en algunas gramáticas también aparece en otras obras, en particular en el Corán y en su comentario (tafșir), en obras de tradición profética, doctrina jurídica y oraciones. El particular sistema de traducción interlineal, palabra por palabra, posibilitaba tener la clave léxica -o glosa- de cada término árabe justamente debajo de éste, permitiendo así el estudio, memorización y comprensión de los textos árabes. Las traducciones interlineales se convertían así en glosarios extremadamente prácticos, y a la vez eran una manera clara de mostrar la sintaxis del árabe. Su función era evidentemente pedagógica.

Hubiera sido más confuso ofrecer la traducción interlineal en caracteres latinos, puesto que, al escribirse este alfabeto de izquierda a derecha, no podría situarse cada palabra traducida debajo de cada término árabe, o, si así se hiciera, no podría leerse con fluidez cada una de las líneas traducidas. Es evidente que el sistema de traducción interlineal o glosa en aljamiado es mucho más sencillo y práctico que un hipotético sistema de traducción interlineal en caracteres latinos.

Esta última constatación sirve de argumento en favor de la hipótesis planteada por Alberto Montaner, para quien la escritura aljamiada podría haber surgido, entre otras posibles causas (hipótesis del origen poligenético), por la necesidad de glosar interlinealmente los textos árabes conservados en las comunidades mudéjares que habían perdido el árabe como lengua mater$\mathrm{na}^{32}$. Así se desarrollaría y perfeccionaría este sistema de escritura durante un período de tiempo más o menos largo, y posteriormente, ya extendido, experimentado y popularizado, saldría de su función estrictamente auxiliar para emplearse independientemente.

32. Véase «El auge de la literatura aljamiada en Aragón», en II Currso sobre lengua y literatura en Aragón (Siglos de Oro), dirigido por Aurora Egido y Tomás Buesa, edición de José $\mathrm{M}^{\mathrm{a}}$ ENGUITA, Zaragoza, 1993, pp. 31-61, esp. 40 y 45. 